\title{
Phenomenological Description of the Acoustic Phonon Branch of $\mathrm{K}_{2} \mathrm{SeO}_{4}$
}

\author{
D. G. SANNIKOV
}

Institute of Crystallography, Russian Academy of Sciences, Moscow 119333, Russia

\section{H. MASHIYAMA}

Department of Physics, Faculty of Science, Yamaguchi University, Yamaguchi 753-8512, Japan

\begin{abstract}
A phenomenological approach is used in investigating dispersion of the acoustic phonon branch of $\mathrm{K}_{2} \mathrm{SeO}_{4}$ in the extended zone scheme. The dispersion is expressed through coefficients of a simple thermodynamic potential. According to symmetry of the crystal $\left(\mathrm{D}_{2 \mathrm{~h}}=\mathrm{mmm}\right)$ the Lifshitz-type invariant exists in the potential. This invariant is responsible for the linear and dynamical interaction of the acoustic and optical branches. The soft optical branch lowering with decreasing temperature forces to lower the acoustic branch which becomes zero at some value of the wave vector.
\end{abstract}

Keywords Phenomenological approach; acoustic branch; $\mathrm{K}_{2} \mathrm{SeO}_{4}$

In the well-known paper by Iizumi et al. [1] the experimental dependence of acoustic branch of $\mathrm{K}_{2} \mathrm{SeO}_{4}$ on a dimensionless wave number is presented in an extended zone scheme (Fig. 1; see also review [2]). Many authors have developed a theory of this dispersion using different theoretical models (see e.g. [3-6]). We consider here a phenomenological approach to the problem, which was not used, as far as we know. This approach is based on the Landau theory of phase transitions, which in turn is based on symmetry considerations.

Our interest is only with the condition of stability loss of the initial (normal) phase. Thus we can present the thermodynamic potential density in the form:

$$
\Phi=\alpha \eta^{2}+\alpha^{\prime} \xi^{2}+\sigma\left(\eta^{\prime} \xi-\xi^{\prime} \eta\right)+\delta \eta^{\prime 2}+\kappa \eta^{\prime 2}+\delta^{\prime} \xi^{\prime 2}+\kappa^{\prime} \xi^{\prime 2} \text {. }
$$

Here only quadratic invariants in two variables $\eta$ and $\xi$ are taken into account. These variables are assumed to be dependent on the dimensionless coordinate $x a^{*}$. The primed $\left(\eta^{\prime}, \xi^{\prime}\right)$ symbols indicate the derivatives of $\eta$ and $\xi$ with respect to $x a^{*}$. The variable $\eta$ transforms according to one of the vector representations of the group $\mathrm{D}_{2 \mathrm{~h}}$, i.e. as the component $P_{\mathrm{z}}$ of the polarization vector $P_{\mathrm{i}}$. The variable $\xi$ transforms according to different one-dimensional representation of the group $\mathrm{D}_{2 \mathrm{~h}}$, as the component $u_{\mathrm{xz}}$ of the strain tensor $u_{\mathrm{ijj}}$. Then $\eta$ is the amplitude of mode which belongs to the optical branch at $q=0$, while $\xi$ belongs to the acoustic branch. Note that we consider the whole branch in 
Fig. 1 as the acoustic branch.

The variables $\eta$ and $\xi$ interact via the Lifshitz-type (LT) invariant (it has the same form as the Lifshitz invariant for two-dimensional representations). The LT-invariant is a characteristic feature of potential (1). To seek the condition of stability loss of the initial phase we write $\eta$ and $\xi$ in the form:

$$
\eta=P_{\mathrm{z}}=\rho_{1} \cos q a^{*} x, \quad \xi=2 u_{\mathrm{xz}} / a^{*}=u_{\mathrm{z}}^{\prime}, \quad u_{\mathrm{z}}=\rho_{2} \sin q a^{*} x
$$

where $u_{\mathrm{z}}$ is the component of the displacement vector $u_{\mathrm{i}}, q$ is the dimensionless wave number.

Substituting Eq. (2) into Eq. (1) and integrating over $x a^{*}$ we obtain the thermodynamic potential in the form:

$$
\Phi=1 / 2\left(\alpha+\delta q^{2}+\kappa q^{4}\right) \rho_{1}{ }^{2}+\sigma \rho_{1} \rho_{2}+1 / 2 q^{2}\left(\alpha^{\prime}+\delta^{\prime} q^{2}+\kappa^{\prime} q^{4}\right) \rho_{2}{ }^{2} .
$$

Minimizing Eq. (3) with respect to $\rho_{1}, \rho_{2}$ and $q$ we arrive at the following expressions for the elastic coefficients $\mathrm{A}_{+-}{ }^{2}$ of the two branches as functions of $q$ :

$$
\begin{gathered}
\mathrm{A}_{+-}{ }^{2} / \mathrm{Y}^{2}=1 / 2\left\{\alpha(q)+\alpha^{\prime}(q)^{+} .\left[\left(\alpha(q)-\alpha^{\prime}(q)\right]^{2}+\left(2 \sigma q^{2}\right)^{2}\right]^{1 / 2}\right\}, \\
\alpha(q)=\alpha+\delta q^{2}+\kappa q^{4}, \quad \alpha^{\prime}(q)=q^{2}\left(\alpha^{\prime}+\delta^{\prime} q^{2}+\kappa^{\prime} q^{4}\right) .
\end{gathered}
$$

The signs +- refer to the optical and acoustic branches, respectively. $\quad \mathrm{Y}$ is a scale factor. The three-term polynomials $\alpha(q)$ and $\alpha^{\prime}(q)$ correspond to the optical and acoustic branches without interaction.

For the purpose of presenting the dependence A. $(q)$ in Fig. 2 at four temperatures as in Fig. 1, the values of coefficients have to be specified. The scale factor Y may be assigned arbitrary. Three coefficients $\alpha^{\prime}, \delta^{\prime}$ and $\kappa^{\prime}$ of the dependence $\alpha^{\prime}(q)$ are determined from the requirement that the acoustic branch has the inclination at small $q$ and a height of the maximum approximately the same as in Fig. 1 and also has the position of the second maximum at $q=1$. The frequency of acoustic branch becomes zero, according to Fig. 1 , at the definite $q=q_{0}=0.69$ and $T=T_{\mathrm{i}}=130 \mathrm{~K}$. The requirement that this would be fulfilled also for the theoretical acoustic branch determines values of two coefficients; we choose $\alpha_{0}$ and $\kappa$. Values of these coefficients must be taken with a high degree of accuracy, see below Eq. (5). As it is usually done in the Landau theory, the thermodynamic potential coefficients are assumed to have the simplest dependence on temperature $T$. Only one coefficient $\alpha$ at the square of the order parameter $\eta$ depends on $T$ linearly: $\alpha=\alpha_{0}+\alpha_{\mathrm{T}}\left(T-T_{\mathrm{i}}\right) / T_{\mathrm{i}}$ ( $T_{\mathrm{i}}$ is the temperature of the stability loss of the initial phase). The coefficient $\alpha_{\mathrm{T}}$ is determined from experimental curves at $T-T_{\mathrm{i}}=15$, $45,120 \mathrm{~K}$. The dispersion of the optical branch is of no interest for us. The point is that we could not identify a soft optical branch among different experimental branches for $\mathrm{K}_{2} \mathrm{SeO}_{4}$. Therefore we put for simplicity the coefficient $\delta=0$. It remains only one coefficient $\sigma$, which we can vary in such a way as to obtain a better agreement between theoretical and experimental dispersions of the acoustic branch.

Thus, values of the coefficients are chosen as follows:

$$
\begin{aligned}
& Y=100, \alpha^{\prime}=3.5, \delta^{\prime}=-2.2, \kappa^{\prime}=0.4, \alpha_{0}=0.247, \\
& \delta=0, \kappa=2.214, \alpha_{\mathrm{T}}=0.15, \sigma=2 .
\end{aligned}
$$


Figure 2 shows the dispersion of acoustic branch, which is computed according to Eqs. (4) and (5). There is agreement between theoretical and experimental dispersions as can be seen from comparison of Fig. 2 with Fig. 1. It should be emphasized that considerable change in the acoustic branch at $T-T_{\mathrm{i}}=45,15,0 \mathrm{~K}$ take place at comparatively small softening of the optical branch: $\mathrm{A}_{+}=54.7,51.4,49.7$ at $q=0$.

To summarize we can say that the existence of the LT-invariant in the thermodynamic potential is the result of symmetry requirements. Due to the LT-invariant the soft optical and acoustic branches interact linearly and dynamically, so that the optical branch lowering with decreasing $T$ pushes downwards the acoustic branch which becomes zero at $q=q_{0}$ and $T=T_{\mathrm{i}}$.

\section{Acknowledgements}

This work was carried out under the Research Program for Special Promotion of the Venture Business Laboratory, Yamaguchi University. One of the authors (D.G.S.) gratefully acknowledges the financial support of the Russian Foundation for Basic Research (Grant No. 03-02-16104).

\section{References}

1. M. Iizumi, J. D. Axe, G. Shirane and K. Shimaoka: Phys. Rev. B 15 (1997) 4392.

2. J. D. Axe, M. Iizumi and G. Shirane: Incommensurate Phases in Dielectrics 2: Materials, R. Blinc and A. P. Levanyuk (eds.), Elsevier, Amsterdam (1986) Ch.10.

3. H. Bilz, H. Buttner, A. Bussmann-Holder, W. Kress, and U. Schroder: Phys. Rev. Lett. 48 (1982) 264.

4. I. Etxebarria, M. Quilichini, J. M. Perez-Mato, P. Boutrouille, F. J. Zuniga and T. Breczewski: J. Phys.: Condens. Matter 4 (1992) 8551.

5. Y. Ishibashi, M. Iwata, and T. Janssen: J. Phys. Soc. Jpn. 70 (2001) 3178.

6. H. Mashiyama and H. Shigematsu: Ferroelectrics 268 (2002) 95. 


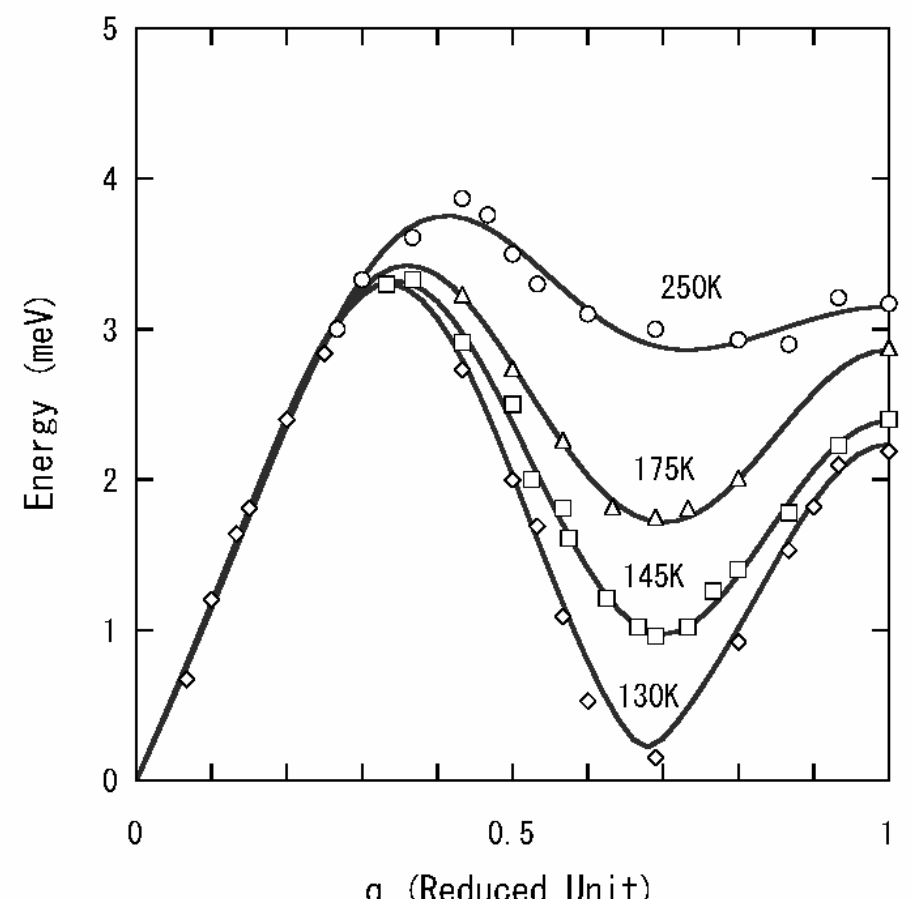

FIGURE 1. Phonon dispersion of the acoustic branch in $\mathrm{K}_{2} \mathrm{SeO}_{4}$ at different temperatures from Ref. [1].

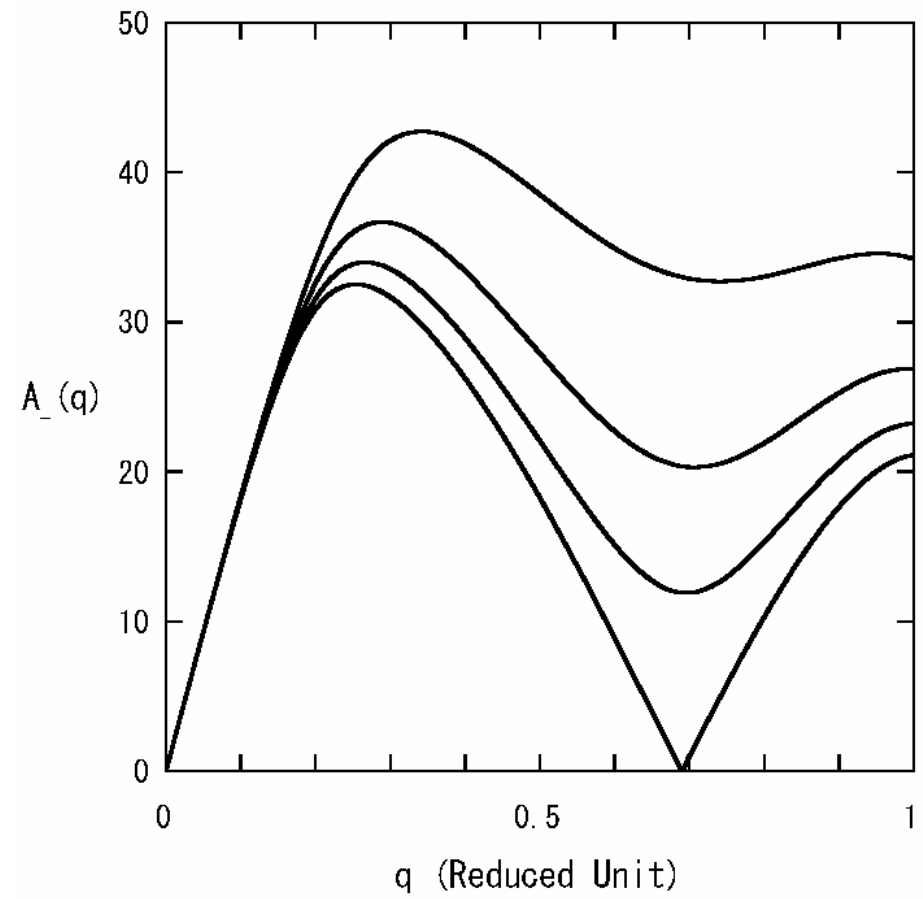

FIGURE 2. Dependence A_( $q$ ) plotted according to Eqs. (4) and (5). 